\title{
Smoke Classification based on curve transform
}

\author{
Tiantian Tang $^{1, \mathrm{a}}$, Linhan Dai ${ }^{1, \mathrm{~b}}$, Zhijian Yin ${ }^{1, \mathrm{c}, ~ *}$ \\ ${ }^{1}$ College of Communication and Electronics, \\ Jiangxi Science \& Technology Normal University, \\ Nanchang, Jiangxi, China. \\ acotang@163.com; ${ }^{b}$ dailinhan9619@163.com; \\ c, *yinzhijian@jxstnu.edu.cn \\ *corresponding author
}

Keywords: Smoke classification, Curvelet transform, Curvelet coefficients, Support Vector Machine

\begin{abstract}
Smoke classification based on video image plays an important role in the performance of fire warning system. This paper presents a smoke classification algorithm based on frequency domain information processing. It is a feature in the video stream image region, which is based on the frequency domain analysis of smoke characteristics. In this paper, a method of extracting features based on Curvelet transform is proposed. Firstly, the multi-scale decomposition of the smoke pattern is preprocessed, and then the effective Curvelet coefficients are extracted as far as possible. At the same time, the selected coefficients as a feature, and finally to the Support Vector Machine classifier to achieve the identification of smoke. The experimental results show that the method can effectively classify the smoke in the frequency domain.
\end{abstract}

\section{Introduction}

Forest fires are one of the most common serious natural disasters, and it tends to cause great harm to human life and property. Currently used for forest fire detection is mainly sensors, such as smoke gas sensors, infrared light sensors, UV sensors, etc., but these sensors in the detection range and anti-interference, there are still many problems. Most of the objects produce smoke before burning. According to this feature, smoke detection can give early warning of fire, so based on the video stream detection method [1] more and more attention. In this paper, we propose a video stream based image processing technology to identify the fire smoke. This paper proposes a feature extraction method based on the Curvelet transform extraction method to identify the fire smoke.

In recent years, wavelet transform has the advantages of good airspace and frequency domain, and has been successfully applied in image noise reduction. However, the advantage of wavelet transform is to reflect the singularity of the signal, that is, it can optimally represent the low-dimensional function with singular singularity, and cannot optimally represent the high-dimensional function with singular singularity or singularity. Therefore, there is a need for a better or "sparse" function representation than wavelet transform to take full advantage of the geometrical properties of the image, that is, multi-scale geometric analysis of images, and Donoho et al [2] proposed Curvelet transformation theory. The first generation of digital Curvelet transform implementation is more complex, requires a series of sub-band decomposition, smooth block, regularization and ridgelet analysis and a series of steps, and the decomposition of the pyramid pyramid also brought a huge amount of data redundancy, Candes et al[3] [4] have proposed a simpler and easier to understand the fast Curvelet transform algorithm, that is, the second generation of the Curvelet (Fast Curvelet transform). Now there are many applications in the second generation of Curvelet transform [5-8].

The second generation of Curvelet transform and the first generation of Curvelet transform in the structure has been completely different. The first generation of Curvelet transform is constructed by 
simply dividing the curve into a straight line in each block by a sufficiently small block, and then using the local ridge to analyze its characteristics, and the second generation of the Curvelet transform and the ridge wave theory There is no relationship, the process does not need to use the ridge, the only point between the two is tight support, framework and other abstract mathematical significance.

\section{Curvelet transform principle}

Continuous ridgelet transform and wavelet transform, ridgelet transform theory are all within the category of sparse theory, using the basis function and the signal (or function) of the inner product form to achieve the signal (or function) is the sparse, and the song transform can be expressed as

$$
\mathrm{c}(\mathrm{j}, \mathrm{l}, \mathrm{k}):=<\mathrm{f}, \varphi_{\mathrm{j}, \mathrm{ll}, \mathrm{k}}>
$$

Among them, $\varphi_{\mathrm{j}, \mathrm{l}, \mathrm{k}}$ represents the coefficient of the curve, $j, l, k$ are the scale, direction, position parameters. The curve transform is implemented in the frequency domain, and the window function $U$ in the frequency domain is used to realize the representation of $\varphi$ in the frequency domain. Define a pair of window functions: radial window function $W(r), r \in\left(\frac{1}{2}, 2\right)$ and angle window function $V(t), t \in[-1,1]$. They all meet the permissible conditions

$$
\begin{array}{r}
\sum_{j=-\infty}^{\infty} W^{2}\left(2^{j} r\right)=1, r \in\left(\frac{3}{4}, \frac{3}{2}\right) \\
\sum_{l=-\infty}^{\infty} V^{2}(t-l)=1, t \in\left(-\frac{1}{2}, \frac{1}{2}\right)
\end{array}
$$

For each $j \geq j_{0}$, the frequency window $U_{j}$ is defined in the frequency domain

$$
U_{J}(r, \theta)=2^{-\frac{3 j}{4}} W\left(2^{-j} r\right) V\left(\frac{2^{[j / 2]} \theta}{2 \pi}\right)
$$

Which [ $j / 2]$ is the $j / 2$ integer part. The support interval of $U_{j}$ is a wedge-shaped region obtained by supporting $W$ and $V$ supporting the spatial limitation, as shown in Fig. 1 for the wedge-shaped shaded area. The wedge region conforms to the anisotropic scale.

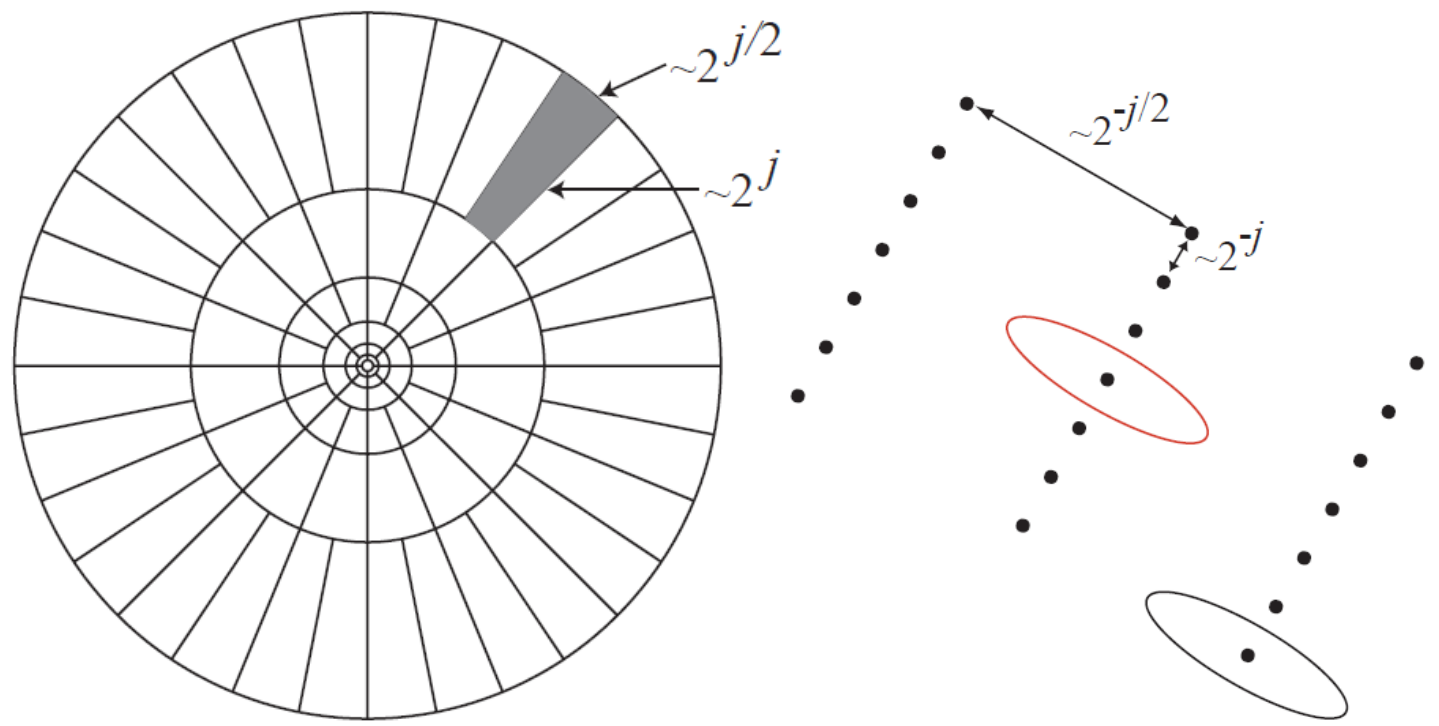

Figure.1. Continuous Curvelet transform transformation frequency space division diagram

The left is in the time domain, the right is in the frequency domain. Defined in the scale of $2^{-j}$, the direction of $\theta_{l}$, translation parameters $\left(k_{1}, k_{2}\right)$ at the continuous curve converted to

$$
\varphi_{j, l, k}(x)=\varphi_{j}\left(R_{\theta_{l}}\left(x-x_{k}^{(j, l)}\right)\right)
$$

$x_{k}^{(j, l)}=R_{\theta_{l}}^{-1}\left(k_{1} \cdot 2^{-j}, k_{2} \cdot 2^{-j / 2}\right), R_{\theta_{l}}$ is obtained by $\theta_{l}$ rotation The Curve transform can be 
expressed as

$$
c(j, l, k):=<f, \varphi_{j, l, k}>=\int f(x) \overline{\varphi_{\jmath, l, k}(x)} d x
$$

By Plancherel theory, the above formula can be deduced

$$
c(j, l, k):=\frac{1}{2 \pi^{2}} \int \hat{f}(\omega) \overline{\varphi_{J, l, k}(\omega)} d \omega=\frac{1}{2 \pi^{2}} \int \hat{f}(\omega) U_{j}\left(R_{\theta_{l}} \omega\right) e^{j<x_{k}^{(j, l)}, \omega>} d \omega
$$

\subsection{Discrete curve changes}

In the Cartesian coordinate system, $f\left[t_{1}, t_{2}\right]\left(0 \leq t_{1}, t_{2}<\mathrm{n}\right)$ is input, and the discrete form of the curve transform is

$$
c^{D}(j, l, k):=\sum_{0 \leq t_{1}, t_{2}<n} f\left[t_{1}, t_{2}\right] \overline{\varphi_{j, l, k}^{D}\left[t_{1}, t_{2}\right]}
$$

Using a pass function $\psi$ ? $\left(\omega_{1}\right)=\sqrt{\phi\left(\omega_{1} / 2\right)^{2}-\phi\left(\omega_{1}\right)^{2}}$, definition

$$
\psi_{j}\left(\omega_{1}\right)=\psi\left(2^{-j} \omega_{1}\right)
$$

Using this function to achieve multi-scale segmentation, for each $\omega=\left(\omega_{1}, \omega_{2}\right), \omega_{1}>0$, there are

$$
\mathrm{V}_{j}\left(S_{\theta_{l}} \omega\right)=V\left(\frac{2^{[j / 2]}}{\omega_{1}}-l\right)
$$

Where $S_{\theta_{l}}$ is a Shear Matrix $S_{\theta_{l}}=\left[\begin{array}{cc}1 & 0 \\ -\tan \theta_{l} & 1\end{array}\right] . \theta_{l}$ is not equidistant, but the slope is equally spaced. Definition

$$
\widetilde{U}_{J}(\omega)=\psi_{j}\left(\omega_{1}\right) V_{j}(\omega)
$$

For each $\theta_{l} \in[-\pi / 4, \pi / 4]$, there are

$$
\widetilde{U_{j,}}(\omega)=\psi_{j}\left(\omega_{1}\right) V_{j}\left(S_{\theta_{l}} \omega\right)=\widetilde{U}_{J}\left(S_{\theta_{l}} \omega\right)
$$

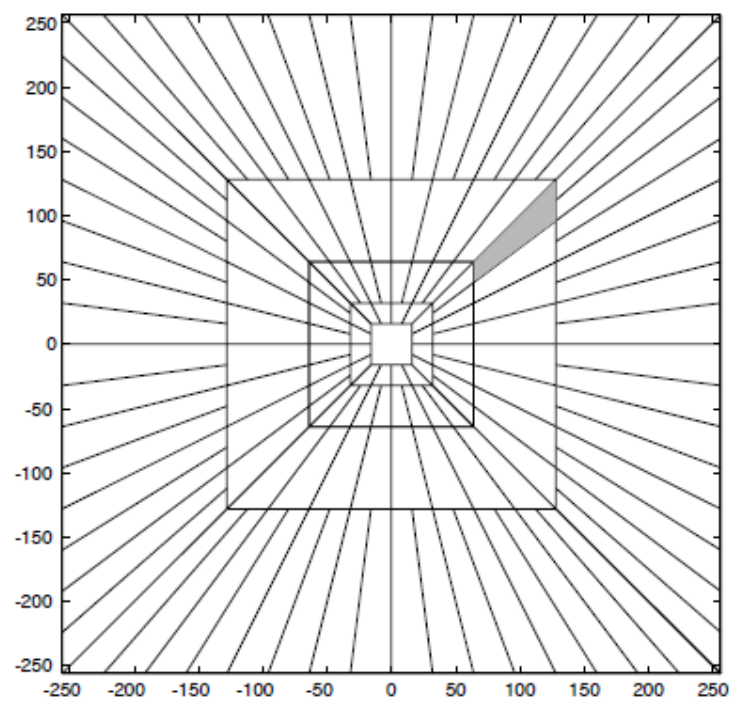

Fig.2. Schematic diagram of frequency division of discrete Curvelet transform

\subsection{Implementation method}

Candes and Donoho proposed two fast discrete curve transform transform methods, namely, USFFT algorithm (unequally-spaced fast fourier transform) and Wrap algorithm (Wrapping-based transform).

Realization of Fast Discrete Curve Transform Based on USFFT

(1) $2 \mathrm{D}$ transform is performed for a given two-dimensional function in a Cartesian coordinate 
system, resulting in a two-dimensional frequency domain representation

$$
\hat{f}\left[n_{1}, n_{2}\right],-n / 2 \leq n_{1}, n_{2} \leq n / 2
$$

(2) In the frequency domain, for each pair ( $\mathrm{j}, \mathrm{l}$ ) (scale, angle), resampling $\hat{\mathrm{f}}\left[n_{1}, n_{2}\right]$, get the sampled value

$$
\hat{f}\left[n_{1}, n_{2}-n_{1} \tan \theta_{l}\right],\left(n_{1}, n_{2}\right) \in P_{j}
$$

Where $L_{1, j}$ is about $2^{j / 2}$ and is the parameter of the length of the support section of the window function $\widetilde{U}_{J}\left[n_{1}, n_{2}\right]$

(3) will be interpolated after the $\mathrm{f}$ and window function $\mathrm{U}$ can be obtained by multiplying

$$
\hat{\mathrm{f}}\left[\mathrm{n}_{1}, \mathrm{n}_{2}\right]=\hat{\mathrm{f}}\left[\mathrm{n}_{1}, \mathrm{n}_{2}-\mathrm{n}_{1} \tan \theta_{\mathrm{l}}\right] \mathrm{U}_{\mathrm{j}}\left[\mathrm{n}_{1}, \mathrm{n}_{2}\right]
$$

(4) The 2D FFT inverse transform of $\hat{f}_{j, l}$ is obtained, and the discrete set of curvature coefficients $c^{D}(j, l, k)$ is obtained.

Realization of Fast Discrete Curve Transform Based on Warpping

The core idea of this method is around the origin of Wrap, meaning that in the specific implementation of any region, through the cyclical technology one by one mapping to the origin of the affine area. The method is based on the USFFT method to increase the wrap step, the specific process is as follows:

(1) 2D FFT transform is performed for a given two-dimensional function in a Cartesian coordinate system, resulting in a two-dimensional frequency domain representation

$$
\hat{f}\left[n_{1}, n_{2}\right],-n / 2 \leq n_{1}, n_{2} \leq n / 2
$$

(2) In the frequency domain, for each pair $(j, l)$ (scale, angle), resampling $\hat{f}\left[n_{1}, n_{2}\right]$, get the sampled value

$$
\hat{f}\left[n_{1}, n_{2}-n_{1} \tan \theta_{l}\right],\left(n_{1}, n_{2}\right) \in P_{j}
$$

(3)It will be interpolated after the $\hat{f}$ and window function $\widetilde{U}$ can be obtained by multiplying

$$
\hat{f}\left[n_{1}, n_{2}\right]=\hat{f}\left[n_{1}, n_{2}-n_{1} \tan \theta_{l}\right] U_{j}\left[n_{1}, n_{2}\right]
$$

(4) around the origin wrapping localized $\hat{\mathrm{f}}\left[n_{1}, n_{2}\right]$

(5) The inverse FFT transform of $\hat{f}_{j, l}$ is obtained, and the discrete set of curvature coefficients $c^{D}(j, l, k)$ is obtained.

\section{Experimental part}

\subsection{Experimental process}

The experiment is roughly divided into three steps:

1) Image preprocessing: Extended image and equalization

The original image size is $48 * 48$ pixels gray-scale smoke image interpolation, get the size of 64 * 64 pixels gray smoke image, and finally the histogram equalization.

2) Fast discrete Curvelet transform

The image obtained in the previous step is subjected to fast discrete Curvelet transform according to the following formula

$$
s=\log (\min (m, n)-3)
$$

Where s represents the number of layers obtained after the Curvelet transform, and $\mathrm{m}$ and $\mathrm{n}$ are 
the sizes of the images subjected to the curve transformation. Thus, different 3-layer scales and coefficients of different directions are obtained, where the specific coefficient structure is shown in Table 1.

Table 1. Coefficient structure

\begin{tabular}{|c|c|c|c|c|c|c|c|}
\hline Layer & scale & The number of orientation & \multicolumn{4}{|c|}{ The form of the matrix } \\
\hline Coarse & $c\{1\}$ & $1(21 * 21)$ & $21 * 21$ & & & & \\
\hline Detail & $c\{2\}$ & $16(4 * 4)$ & & $18 * 22$ & $16 * 22$ & $22 * 18$ & $22 * 16$ \\
\hline Fine & $c\{3\}$ & $1(64 * 64)$ & $64 * 64$ & & & & \\
\hline
\end{tabular}

3) SVM classification

SVM selection is RBF nuclear classification, the kernel function of the formula is as follows:

$$
\mathrm{k}(\mathrm{x}, \mathrm{y})=\exp \left(-\frac{\|x-y\|^{2}}{2 r^{2}}\right)
$$

Where $\mathrm{r}$ is the parameter of the kernel function.

\subsection{Experimental results}

The experimental smoke dataset is downloaded via the web: http://sit.jxufe.cn/yfn/vsd.html. The training set consists of 1383 images, including 552 smoke images and 831 non-smoke images; the test set consists of 1505 images, including 688 smoke images and 817 non-smoke images.

For the experimental results, as shown in Table 2. There are three standard evaluation indicators, namely, Detection Rate (DR), False Alarm Rate (FAR), and Error Rate (ERR) .An prefer detection method should have high DR, low FAR and ERR at the same time.

The coefficients of the curve and the wavelet transform of the smoke image are shown in Fig. 3 and Fig.4.

Table 2. Experimental result

\begin{tabular}{cccc}
\hline \multirow{2}{*}{ methods } & \multicolumn{3}{c}{ smoke dataset } \\
\cline { 2 - 4 } & DR $(\%)$ & FAR $(\%)$ & ERR $(\%)$ \\
\hline Curvelet & 79.36 & 16.401 & 18.339 \\
\hline Wavelet & 59.593 & 19.951 & 29.302 \\
\hline
\end{tabular}

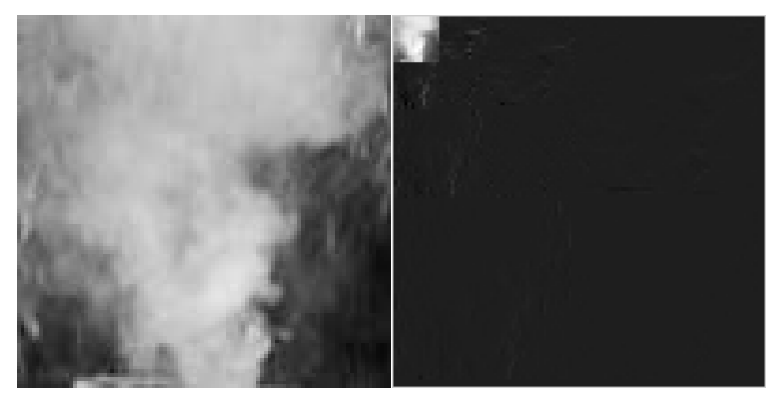

Figure 3. Example of wavelet coefficients: Original image (left) 3-layer wavelet(right).

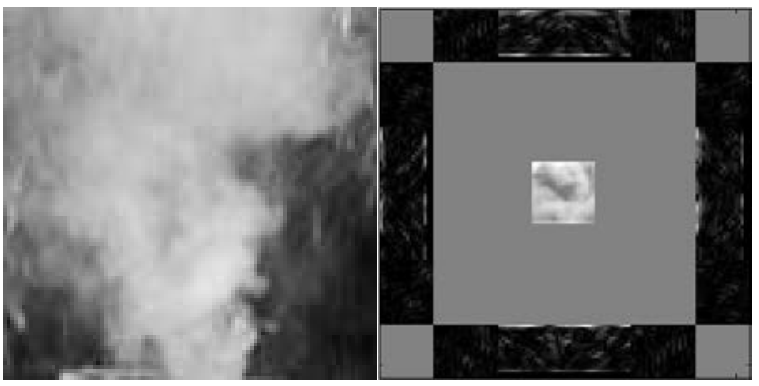

Figure 4. Example of Curvelet coefficients: Original image (left) Coefficients (right, scale=3). 


\section{Conclusion}

Based on the second generation of the Curvelet transform, the method of extracting the feature of the smoke image in the frequency domain and combining the SVM classification method can better extract the edge detail information of the image, increase the feature information of the image, and make the final classification So well prepared. Compared with the wavelet transform method, only a small amount of edge information and feature information can be extracted, resulting in low detection of classification. However, the dimensionality of the feature extracted in this method is too high, resulting in a longer time for the classification, and the next step should be to reduce the feature dimension. The second generation of curved wave transform has a wide application prospect in image processing field as a new multi - scale geometric analysis tool.

\section{Reference}

[1] Yuan, F., Video-based smoke detection with histogram sequence of LBP and LBPV pyramids. Fire Safety Journal, 2011. 46(3): p. 132-139.

[2] Ma, J. and G. Plonka, The Curvelet Transform. IEEE Signal Processing Magazine, 2010. 27(2): p. 118-133.

[3] Candès, E.J. and D.L. Donoho, Continuous curvelet transform : II. Discretization and frames. Applied \& Computational Harmonic Analysis, 2005. 19(2): p. 198-222.

[4] Emmanuel Cand`es†, L.D., David Donoho] and Lexing Ying, Fast Discrete Curvelet Transforms. Society for Industrial and Applied Mathematics, 2006.

[5] Jiulong, Z., et al., Face recognition based on curvelet and PCA. Computer Application, 2008. 28(5): p. 1255-1256.

[6] XianXing, W., et al., Face processing in human-computer Interaction using curvelet analysis. Journal of Image and Graphics, 2010. 15(9): p. 1309-1317.

[7] Yunqiang, Z., et al., Invariant moment extraction by curvelet transform for wear particle images. Journal of Image and Graphics, 2012. 17(2): p. 110-115.

[8] Candes, E. The curvelet transform for image denoising. in International Conference on Image Processing, 2001. Proceedings. 2002. 